\title{
Computational Model and Numerical Simulation for Submerged Mooring Monitoring Platform's Dynamical Response
}

\author{
Kongde $\mathrm{He}^{1,2}$ Zifan Fang ${ }^{1,2}$ Weihua Yang ${ }^{1,2}$ \\ (1. College of Mechanical and Power Engineering, China Three Gorges University, Yichang 443002) \\ (2. Hubei Provincial Collaborative Innovation Center for New Energy Microgrid, China Three Gorges University)
}

\begin{abstract}
Computational model and numerical simulation for submerged mooring monitoring platform were formulated aimed at the dynamical response by the action of flow force, which based on Hopkinson impact load theory, taken into account the catenoid effect of mooring cable and revised the difference of tension and tangential direction action force by equivalent modulus of elasticity. Solved the equation by hydraulics theory and structural mechanics theory of oceaneering, studied the response of buoy on flow force. The validity of model were checked and the results were in good agreement; the result show the buoy will engender biggish heave and swaying displacement, but the swaying displacement got stable quickly and the heaven displacement cause vibration for the vortex-induced action by the flow.

Key words: Submerged mooring monitoring platform; Fluid-solid coupling; Calculation model; Numerical simulation
\end{abstract}

\section{Introduction}

The submerged mooring monitoring platform was a support platform for equipments and instruments of image acquisition for submerged monitoring system, which transverse fixed on security region of river, the sonar on it was vertical and up to water, which could continuously emit pulse, so it could scan the security region which need scanned, and the sonar transmit received echo data to sonar processor which seated on river bank, formed singlewidth narrow-band image, which was handled by sonar processor and formed three dimensional image to identified. The submerged mooring monitoring platform, information processing and recognition system of base station and emergency treatment system composed monitoring system.

it was formed by anchor, mooring cable, buoy, sonar and sensors which fixed on buoy. This equipment need work in deep water for a long time and it could measure lots of environment parameters for water area around continuously and real-time. In order to obtain real-time and stability signal, studied the dynamical response which caused by complicated outer loads for buoy was great significance

The submerged platform was a new-style monitoring equipment and had no existing design specifications and data to reference. Its working environment was complicated and it bear lots of uncertainly loads. Except the gravity was certainly load, the wave load, flow load were uncertainly loads, the randomness of all loads must be considered on design and analysis for buoy. Although few scholars at home and abroad were engaged in study the submerged

Email:hekongde@ctgu.edu.cn mooring monitoring platform, but some scholars were engaged in study the marine mooring platform and submerged floating tunnel and obtained some achievements, the achievements had certain referenced significance for submerged mooring monitoring platforms design. Kamman and Huston studied and simulated the variable length and fixed length cable drag system by space discrete method [1], Niedzwecki and Thampi put forward a kind of lumped mass model for multiple degree of freedom to study the response of submerged cable on impact loads, but this method had large calculated quantity and the precision for dealing with the elasticity of cable was not very good [2]. Xiaoping $\mathrm{Li}$ and other scholars found the dynamical equation of three dimensional finite element model for submerged cable by the finite element method put forward by Huston, considered the properties of flow field, deduced the expression of tangential force, normal resistance and added mass of fluid, but they didn't consider the mutational load and the vibration generated by coupling effect of cables and buoy [3]. Jutin Mai studied the dynamical response of submerged tunnel under the action of wave flow and reduced the mechanical model to a simply supported beam on two ends, but the model style was not the same as the submerged mooring monitoring platform[4]. The computation model and numerical model of submerged mooring monitoring platform was formed by considering the impact loads and referenced the Hopkinson impact load theory, respectively solved the equation by analytical method and numerical simulation method based on hydraulics theory and structural 
mechanics theory of oceaneering, studied the dynamical response of buoy by example.

\section{Analytical solution}

According to the motion characteristics of buoy and the interaction between mooring cable and buoy, the main factors influenced buoy moving was the plane motion in the direction of flow velocity; its simplified schematic plot was shown as Fig1.

The buoy would emerge instability motion and its initial balance was destroyed when hydrological environment change or meet the extraneous interference. The mooring cable would emerge abrupt tighten or slack by outer interference for its light weight, impact was generated between buoy and mooring cables and impact load was generated too. According to the Hopkinson impact theory[5], because buoy move in YOZ plane, when buoy move suddenly, the impact force would generate between buoy and mooring cable, the elastic tension would emerge too for the elasticity of mooring cables, at the same time, the buoy received the drag force along flow direction, resistance of flow, buoyancy and gravity. The origin of coordinate $O$ was set on the initial barycentre of buoy; ignored wave force, according to Newton's law of motion, dynamical equation when buoy and mooring cable impact were found as below:

$$
\begin{aligned}
& m \frac{d^{2} Y}{d t^{2}}=-A_{c} \rho_{c} C_{0} \frac{d Y}{d t}-k Y-C \frac{d Y}{d t}+F_{Y} \\
& m \frac{d^{2} Z}{d t^{2}}=-A_{c} \rho_{c} C_{0} \frac{d Z}{d t}-k Z-C \frac{d Z}{d t}+F_{Z}
\end{aligned}
$$

$m$ was quality of buoy, $A_{c}$ was cross section area of mooring cable, $\rho_{c}$ was density of mooring cable, $C_{o}=\sqrt{E / \rho_{c}}, E$ was modulus of elasticity for mooring cable, $k$ was stiffness coefficient of mooring cable, $C$ was damping coefficient of hydrodynamic force.

The initial displacement and velocity of buoy would emerge after impact, the buoy would move continue according to the initial displacement as starting point, initial velocity as original speed, in the moving process, the buoy received the drag force along flow direction, resistance of flow, buoyancy and gravity, at the end of moving, buoy will got new equilibrium position, according to Newton's law of motion, dynamical equation after buoy and mooring cable impact were found as below:

$$
\begin{aligned}
& m \frac{d^{2} Y}{d t^{2}}=-k Y-C \frac{d Y}{d t}+F_{Y} \\
& m \frac{d^{2} Z}{d t^{2}}=-k Z-C \frac{d Z}{d t}+F_{Z}
\end{aligned}
$$

The force diagram of buoy on equilibrium position was shown as Fig.2:

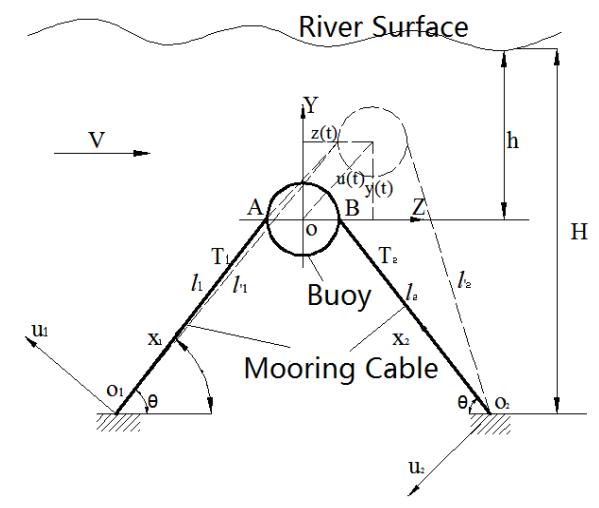

Fig.1 Simplified schematic plot of monitoring platform

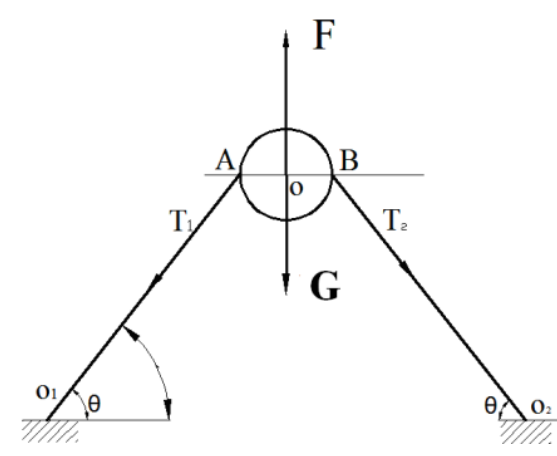

Fig.2 Force diagram of buoy on static balance position

The buoy received the tension of mooring cable $T_{1}$, $T_{2}$, buoyancy $F$ and gravity $G$ on equilibrium position. The mooring cables were fixed symmetrical, so:

$$
T_{1}=T_{2}=\frac{F-G}{2 \sin \theta}
$$

$\theta$ was the fixed angle of mooring cable, according to material mechanics principle, the distortion of mooring cable with the action of $T_{1}, T_{2}$ was:

$$
\Delta l=\frac{T_{1} l}{E A}
$$

Where $l$ was the length of mooring cable.

a) Tension and distortion of mooring cable when buoy moving

1) Distortion of mooring cable

The second item on the right of formula (1), (2), (3), (4) was tension of mooring cable for taking the elastic deformation account. Its value was product of stiffness and distortion of mooring cable. The distortion for mooring cable on both sides of the buoy was different because the buoy received the flow force on one side. According to Fig.3 and deformation compatibility condition of displacement, the elastic distortion of mooring cable for moving was supposed as $u_{i}(t)$.

$$
u_{1}(t)=l_{1}^{\prime}-l_{1}=Y \cos \theta+Z \sin \theta
$$




$$
u_{2}(t)=l_{2}^{\prime}-l_{2}=Y \cos \theta-Z \sin \theta
$$

\section{2) Stiffness of mooring cable}

The mooring cable was the main constraint component for submerged mooring monitoring platform; its performance significantly influenced the dynamical property of buoy. The mooring cable was a flexible member which can only receive one-way tensile stress, it would emerge a certain vertical degree by gravity and flow force, so vertical degree couldn't be omitted when calculating Stiffness of mooring cable. At present equivalent elastic modulus method was the main method to calculate the vertical degree effect. Because the mooring cable under the water was in tighten state, during analysis, its tension was regarded along chordwise, but the mooring cable had certain vertical degree, the tension and force of chordwise was different, according to Ernst's advice, the deviation could be revised by equivalent elastic modulus method [6], the formula was:

$$
E_{e q}=\frac{E}{1+\frac{\left(q l_{x}\right)^{2} E A_{c}}{12 T^{3}}}
$$

$q$ was unit length gravity of mooring cable, $l_{x}$ was horizontal project length, $T$ was initial tension of mooring cable, according to material mechanics principle, $k=\frac{E_{e q} A_{c}}{l}$.

3) Tension of mooring cable when moving

The force on $Y$ direction of mooring cable was below.

$$
k\left[u_{1}(t)+u_{2}(t)\right] \sin \theta=2 k Y \sin \theta \cos \theta
$$
below.

The force on $Z$ direction of mooring cable was

$$
k\left[u_{1}(t)+u_{2}(t)\right] \cos \theta=2 k Y \cos ^{2} \theta=2 k Z \frac{\cos ^{3} \theta}{\sin \theta}
$$

b) Flow force

The origin of coordinate $O$ was set on initial barycentre of buoy, $F_{Y}$ was resultant force for vertical lift force $F_{L}$ of flow and initial force $F_{I Y}$ along $Y$ direction; $F_{Z}$ was resultant force for drag force $F_{d}$ of flow and initial force $F_{I Z}$ along $Z$ direction; $F_{d}$ could be calculated according to Morison formula[7], taking into account relative motion for buoy and flow, supposed river water was uniformity steady-state flow.

$$
\begin{array}{r}
F_{d}=C_{D} \rho \frac{D}{2}\left|v-\frac{d Z}{d t}\right|\left(v-\frac{d Z}{d t}\right) L \\
F_{I Y}=C_{M} \rho \pi \frac{D^{2}}{4} \frac{d^{2} Y}{d t^{2}} L
\end{array}
$$

$C_{D}$ was viscosity damping coefficient; $\rho$ was density of water; $D$ was diameter of buoy; $v$ was velocity of water; $C_{M}$ was initial force coefficient; $m_{0}$ was unit length gravity of buoy; $L$ was length of buoy.
$F_{L}$ Can be calculated according to "Karman's vortex" theory:

$$
\begin{gathered}
F_{L}=C_{L} \frac{1}{2} \rho v^{2} D L \\
F_{I Z}=C_{M} \rho \pi \frac{D^{2}}{4} \frac{d^{2} Z}{d t^{2}} L
\end{gathered}
$$

Where $C_{L}$ was coefficient of lift.

c) Damping coefficient of hydrodynamic force

According to reference[8], damping coefficient of hydrodynamic force $C$ could be calculated according to formula below:

$$
C \approx \frac{4 \omega}{3 \pi} \rho C_{D} A_{L} U
$$

Where $\omega$ was undamped nature frequency; $A_{L}$ was cross section area of buoy; $U$ was amplitude of state response for mooring cable.

\section{d) Dynamical equation after buoy and mooring} cable impact

In respect the number of mooring cable was three double, put formula (5) (16) into (1), (2), (3), (4), dynamical equation when buoy and mooring cable impact could be got as below:

$$
\begin{gathered}
\left(m+C_{M} \rho \pi \frac{D^{2}}{4} L\right) \frac{d^{2} Y}{d t^{2}}+\left(6 A_{c} \rho_{c} C_{0}+C L\right) \frac{d Y}{d t} \\
+6 k \sin \theta \cos \theta Y=C_{L} \frac{1}{2} \rho v^{2} D L+(F-G) \\
\left(m+C_{M} \rho \pi \frac{D^{2}}{4} L\right) \frac{d^{2} Z}{d t^{2}}+\left(6 A_{c} \rho_{c} C_{0}+C L\right) \frac{d Z}{d t} \\
+6 k \frac{\cos ^{3} \theta}{\sin \theta} Z=C_{D} \rho \frac{D}{2}\left|v-\frac{d Z}{d t}\right|\left(v-\frac{d Z}{d t}\right) L
\end{gathered}
$$

Dynamical equation after buoy and mooring cable impact could be got as below:

$$
\begin{gathered}
\left(m+C_{M} \rho \pi \frac{D^{2}}{4} L\right) \frac{d^{2} Y}{d t^{2}}+C L \frac{d Y}{d t} \\
+6 k \sin \theta \cos \theta Y=C_{L} \frac{1}{2} \rho v^{2} D L+(F-G) \\
\left(m+C_{M} \rho \pi \frac{D^{2}}{4} L\right) \frac{d^{2} Z}{d t^{2}}+C L \frac{d Z}{d t} \\
+6 k \frac{\cos ^{3} \theta}{\sin \theta} Z=C_{D} \rho \frac{D}{2}\left|v-\frac{d Z}{d t}\right|\left(v-\frac{d Z}{d t}\right) L
\end{gathered}
$$

Where:

$$
K=\frac{E(F-W)^{3} A_{c} \sin \theta}{18 q^{2}(H-h)^{3} \sin \theta \cos ^{2} \theta E A_{c}+(F-W)^{3}(H-h)}
$$

\section{Impact time of buoy and mooring cable}

According to reference[9], when action time was less than half of natural period of vibration for impacted body, the action load could be called impact load, impact time could be calculated according to below method, taking into account the moment of impact, 
mooring cable will move with buoy action, the period of motion for buoy restrained by mooring cable was $2 \pi \sqrt{\frac{m l}{E A_{c}}}$, so impact time was:

$$
\Delta t=\pi \sqrt{\frac{m l}{E A_{c}}}
$$

\section{Vertical velocity distribution character of hydrology section for Yangtze River}

The location fixed submerged mooring monitoring platform was chose Huanglingmiao water area of Yi Chang, according to the statistical data of hydrometric station, the vertical velocity distribution character of the area was accord with Karaueshev's ellipse flow velocity distribution rule[10].

$$
v=v_{0} \sqrt{1-p \eta^{2}}=\sqrt{p} v_{0} \sqrt{\frac{1}{p}-\eta^{2}}
$$

Where: $v_{0}$ was velocity of water surface $(\eta=0)$; $p$ was distributed parameters of flow velocity, $p=0.6$; $\eta$ was relative depth of water from water surface.

The vertical mean flow rate $v_{m}$ could be calculated with integration:

$$
v_{m}=\int_{0}^{1} v d \eta=\sqrt{p} v_{0} \int_{0}^{1} \sqrt{\frac{1}{p}-\eta^{2}} d \eta=0.897 v_{0}
$$

According to Fig.3, the velocity of water at the buoy position was below:

$$
v=\frac{v_{m}}{0.897} \sqrt{1-0.6\left(\frac{h}{H}\right)^{2}}
$$

When study the project, hydraulic factors and grain diameters of bed material at the section Huanglinmiao were tested, the test result was shown as Tab.1.

Tab.1 Hydraulic factors and grain diameters of bed material at the section Huanglinmiao

\begin{tabular}{llll}
\hline $\begin{array}{l}\text { Number of viewing } \\
\text { perpendicular }\end{array}$ & $1^{\#}$ & $2^{\#}$ & $3^{\#}$ \\
\hline Water temperature $\left({ }^{\circ} \mathrm{C}\right)$ & 23.2 & 23.8 & 22.4 \\
Distance to start point $(\mathrm{m})$ & 510 & 350 & 310 \\
Viewing time interval (s) & 2.27 & 2 & 0.57 \\
Rate of flow $\left(\mathrm{m}^{3} / \mathrm{s}\right)$ & 30000 & 42200 & 11200 \\
Depth of water(m) & 17.1 & 63.2 & 60.87 \\
Discharge section area(m $\left.{ }^{2}\right)$ & 17922 & 18530 & 17092 \\
Width of river surface $(\mathrm{m})$ & 504 & 511 & 495 \\
Gradient of water surface $(\%)$ & 0.102 & 0.181 & 0.019 \\
\hline
\end{tabular}

From Tab.1, the water flow of No. $2^{\#}$ was maximum, water depth was maximum, mean flow rate was $2.27 \mathrm{~m} / \mathrm{s}$, so No. $2^{\#}$ was chose as the fixed position for mooring monitoring platform when study the project.

\section{Numerical simulation}

\subsection{Model}

For checking the result of computation, the fluidsolid coupling method of Msc/Nastran software was used to simulate the submerged mooring monitoring platform's motion process. Because the buoy was cylindrical and thin shell body, it was welded by steel tub, so according to properties for endurance of buoy, we chose $2 D$ shell type element when construct the finite element model; and according to equivalent elastic modulus method, mooring cable was simulated by Rod type element, the fluid-solid coupling model was founded as Fig.3 and Fig.4. The flow force was dealt with virtual mass ${ }^{[1]}$.

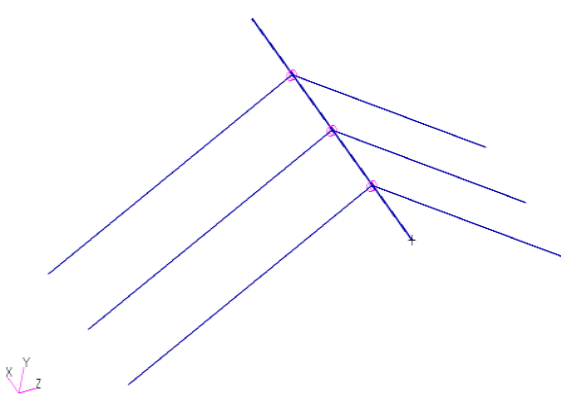

Fig.3 Finite element model

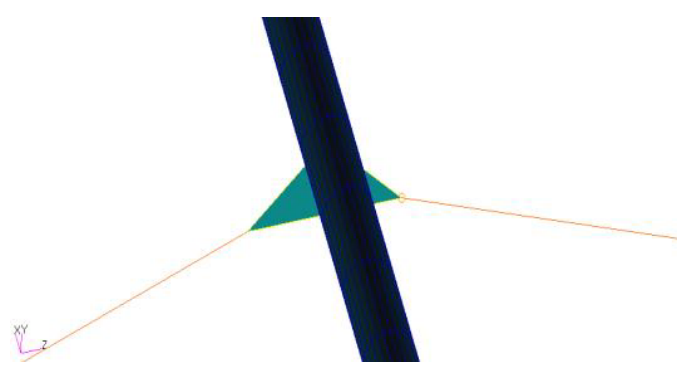

Fig.4 Partial enlarged drawing of Finite element model

\subsection{Fluid-solid coupling face}

The aim for defining fluid-solid coupling face was make the material on Euler element (water) and Lagrange element (buoy) acts each other. If the relation of coupling did not define, even if the Lagrange element just surrounded by Euler element, the force made by Lagrange element couldn't effect the Euler element, and simultaneity the Lagrange element itself didn't endure the force made by Euler element. For constructing the coupling relation of buoy and water, first the coupling face on buoy must be defined, through the face, the buoy and water can act to each other. For water, the face was the boundary of fluid. And at the same time, the force caused by fluid can act on coupling face, make the deformation of buoy, the coupling face sketch map was as show in Fig.5:

When we analyzed fluid-solid coupling, the coupling face must be closed and have positive volume, so it required all the directions of normal line point to outer side, as show in Fig.6. Further more, the closed 
coupling face must interact with at least one Euler element, or, the coupling couldn't take place.

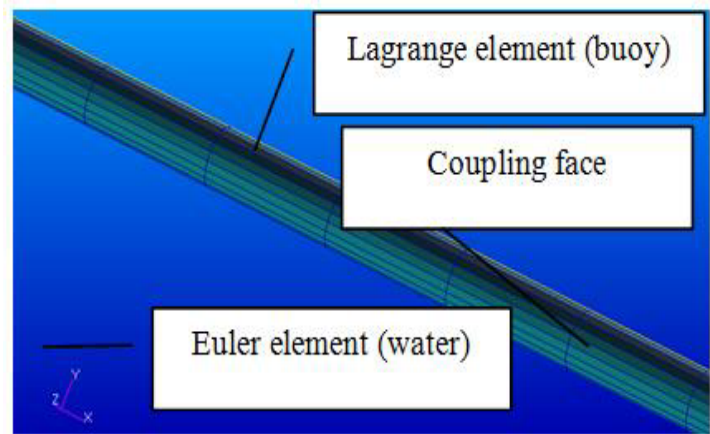

Fig.5 Coupling face of water and buoy

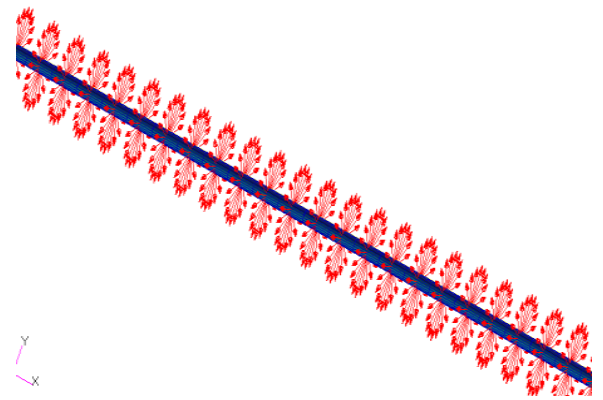

Fig.6 Closed coupling face

\section{Example}

\subsection{Design parameters}

According to design requirements and above analysis, related parameters were shown as Tab.2.

Tab.2 Design parameters

\begin{tabular}{|c|c|c|c|}
\hline $\begin{array}{c}\text { Length of buoy } \\
L(\mathrm{~m})\end{array}$ & 50 & $\begin{array}{c}\text { Coefficient of initial } \\
\text { force } C_{M}\end{array}$ & 1 \\
\hline $\begin{array}{c}\text { Diameter of buoy } \\
D(\mathrm{~m})\end{array}$ & 0.2 & $\begin{array}{c}\text { Density of river } \\
\rho\left(\mathrm{kg} / \mathrm{m}^{3}\right)\end{array}$ & $1 \times 10^{3}$ \\
\hline $\begin{array}{c}\text { Distance of } \\
\text { mooring cable } l(\mathrm{~m})\end{array}$ & 12.5 & Mass of buoy $(\mathrm{kg})$ & 1463 \\
\hline $\begin{array}{c}\text { Modulus of } \\
\text { elasticity for buoy } \\
E(\mathrm{pa})\end{array}$ & $2 \times 10^{11}$ & $\begin{array}{c}\text { Number of mooring } \\
\text { cable }\end{array}$ & 6 \\
\hline \multicolumn{2}{|c|}{} & \multicolumn{2}{|c|}{} \\
\hline
\end{tabular}

\subsection{Analytic solutions}

Put design parameters into formula (21), impact time could be calculated as: $\Delta t=0.718 \mathrm{~s}$.

Put design parameters into formula (17), (18), the displacement curve of $Y, Z$ direction when impact could be got below as Fig.7 and Fig.8.

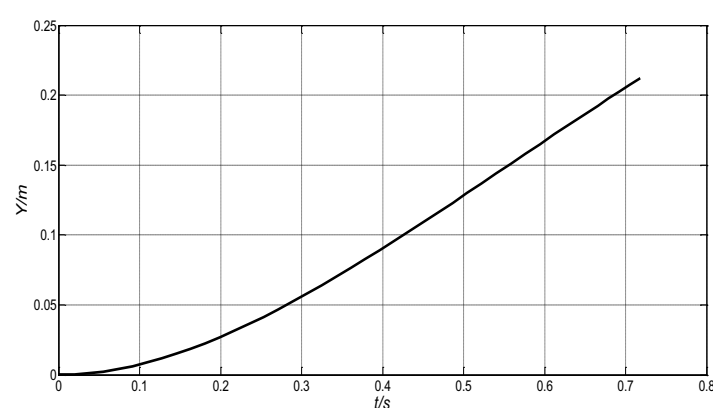

Fig.7 The curve of Y direction displacement of buoy on impact

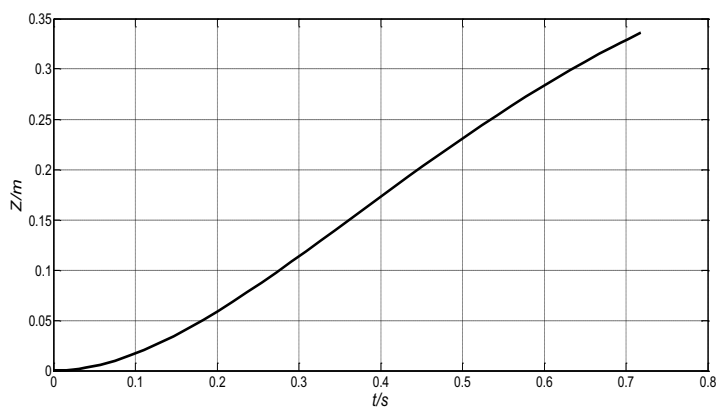

Fig.8 curve of $Z$ direction displacement of buoy on impact

From Fig7, Fig8, the conclusion can be got the displacement of $Y$ direction is $0.29 \mathrm{~m}$ and $Z$ direction is $0.33 \mathrm{~m}$ at impact end.

Formula (19), (20) can be calculated with new boundary conditions which was displacement and velocity at the end of impact, so the response of buoy at the end of impact to stability could be got as Fig.9 and Fig. 10 .

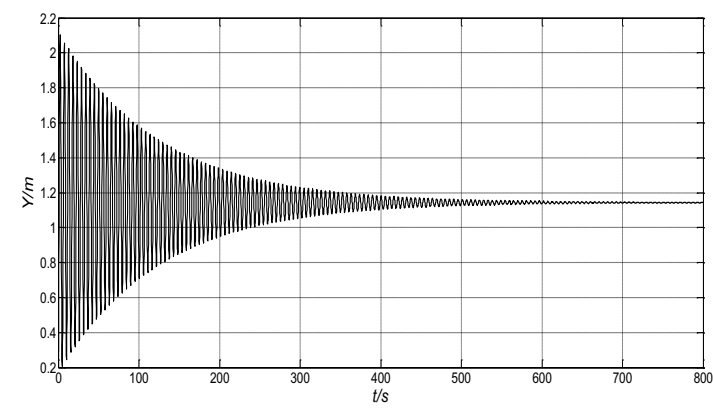

Fig.9 The curve of Y direction displacement of buoy after impact

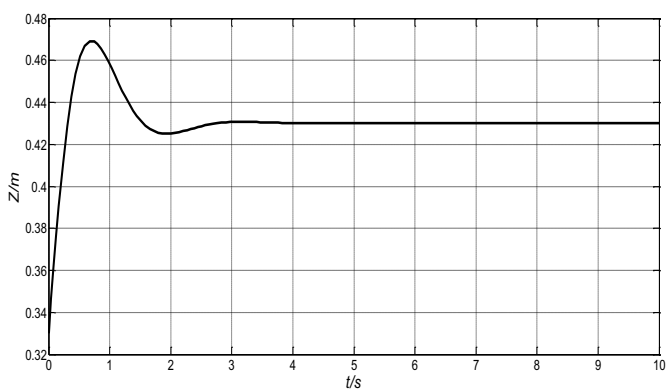

Fig.10 curve of $Z$ direction displacement of buoy after impact

From Fig.9 and Fig.10, the conclusion can be got buoy will move continue at the end of impact, because it still 
received drag force, lift force and buoyancy of flow, at last it got new stable position for damping force of water, but along the flow direction buoy got stable quickly and perpendicular to flow velocity buoy vibrate by vortex lift. At the end of stability state, the displacement of $\mathrm{Y}$ direction is about $1.14 \mathrm{~m}$ and $\mathrm{Z}$ direction is $0.43 \mathrm{~m}$.

\subsection{Numerical solution}

The fluid-solid coupling model was solved, the displacement response for buoy in $\mathrm{Y}, \mathrm{Z}$ direction was shown as Fig.11, Fig.12.

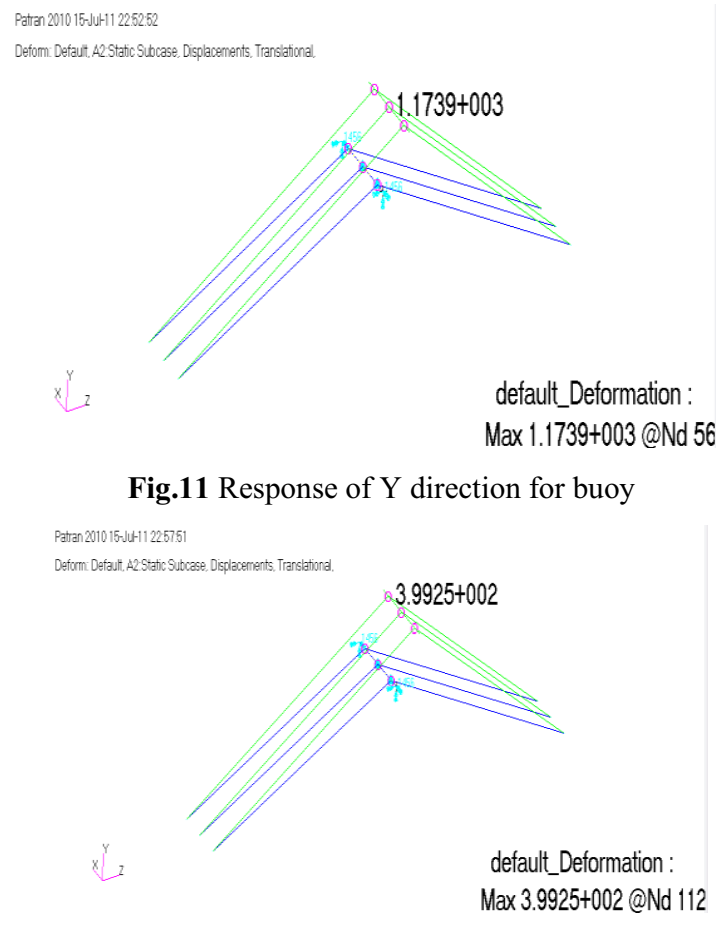

Fig.12 Response of $Z$ direction for buoy

Compared computational solutions with numerical simulation solutions, the result were shown as Tab.3.

Tab.3 Comparison of computational solutions and numerical solution

\begin{tabular}{|c|c|c|}
\hline & $\begin{array}{c}\text { Displacement in Y } \\
\text { direction }(\mathrm{m})\end{array}$ & $\begin{array}{c}\text { Displacement in Z } \\
\text { direction }(\mathrm{m})\end{array}$ \\
\hline $\begin{array}{c}\text { Computational } \\
\text { solutions }\end{array}$ & 1.14 & 0.43 \\
\hline $\begin{array}{c}\text { Numerical } \\
\text { solutions }\end{array}$ & 1.17 & 0.399 \\
\hline Error & $2.6 \%$ & $7.2 \%$ \\
\hline
\end{tabular}

From Tab.3 the conclusion can be got the computational solutions and numerical solutions' error for displacement on $\mathrm{Y}$ direction is $2.6 \%$ and on $\mathrm{Z}$ direction is $7.2 \%$. The reason for error was that when solved the computational solutions the buoy's model founded as rigid body, but when solved the numerical solutions the buoy's model founded as finite element model, the buoy was flexible body, and take into account the self deformation of buoy with mooring cables constraint, so numerical solutions was more accurate and reliability.

\section{Conclusions}

1) The submerged mooring monitoring platform would emerge biggish sway and heave displacement by drag force, lift force and buoyancy of flow, but along the flow direction buoy get stable quickly and perpendicular to flow buoy vibrate by vortex lift.

2) The result of numerical model and computational model for considering the fluid-solid coupling action was anastomotic very well, but there were errors. the reason for error was that when solved the computational solutions the buoy's model founded as rigid body, but when solved the numerical solutions the buoy's model founded as finite element model, the buoy was flexible body, and taken into account the self deformation of buoy with mooring cables constraint, so numerical solutions was more accurate and reliability.

3) The Influence caused by vortex lift couldn't be omitted when further study the stability of submerged mooring monitoring platform.

\section{Acknowledgement}

This study was supported by the natural science foundation of Hubei Province project(2015CFB559), (2013CFA132); the educational commission of Hubei Province project (D20141202); The scientific fund of China Three Gorges University project (KJ2013B042).

\section{References}

1. Kamman, James W, Huston, Ronald L. Modeling of variable length towed and tethered cable systems computational[J]. Journal of Guidance, Control and Dynamics, 1999, 22 (4) :602-608.

2. Niedzwecki, John, Thampi, Sreekumar K. Snap loading of marine cable systems[J]. Applied Ocean Research, 1991, 13(S) :210-218.

3. XiaoPin Li. Multi-body Dynamics Modeling and Its Application in Marine Cable Systems [D]. Tian Jin: Tian Jin university, 2004.

4. Jutin Mai. The Study on Responses of a Submerged Floating Tunnel Subjected to the Wave and Current [D].Chen Du: southwest Jiaotong University, 2005.

5. Xiaoqin Ma. Impact Dynamics[M], Press of Beijing Institute of Technology, 1992.

6. Pao-Hsii Wang and Chiung-Guei Yang. Parametric studies on cable-stayed bridges and structures[J], 1996, 60(2):243-260

7. Yougang Tang. Structure Mechanics of Oceaneering [M], Press of Tianjing University, 1992.

8. Dongyao Wang, Guocan Lin. Vortex-induced nonlinear vibrations of TLP tethers under circumstances of platform oscillation $[\mathrm{J}]$. Journal of Ocean. 1998, 20(3):119-128. 
9. Bangguo Lin. High Precision Measurement of Collision Time and Its Application [J]. Journal of Nan Tong Engineering College, 2002, 1(3):4-6.

10. ShiMin Yao, Junyou Lu,Haitao Xu. Study of Vertical Velocity Distribution at Huanglingmiao Hydrologic Section [J], Journal of Yangtze river academy of sciences, 2005, 22(4): 9-11.

11. Jian Yang. MD Nastran finite element Example course[M], China Machine Press, 2008. 